\title{
A numeric far field model for support interference studies in a slotted wall wind tunnel (European Transonic Windtunnel)
}

\author{
A Heidebrecht \\ Institute of Aerodynamics and Flow Technology, DLR Braunschweig, Lilienthalplatz 7, Braunschweig 38108, Germany. \\ email: alexander.heidebrecht@dlr.de
}

The manuscript was received on 9 February 2006 and was accepted after revision for publication on 6 June 2006.

DOI: 10.1243/09544100JAERO92

\begin{abstract}
This article presents a simplified computational fluid dynamics (CFD) model of the European Transonic Windtunnel test section that represents the model support in a far field and is specifically designed for the investigation of model support effects in the transonic regime. Two parametric studies were carried out in order to determine geometric parameters of the adapted support geometry and in order to enable the simulation of actual wind tunnel experiments, by correlating the wind tunnel reference Mach number with that at the far field in the CFD computation and the effective Mach number at the model location. A comparison was done using measured calibration data from the wind tunnel.
\end{abstract}

Keywords: transonic wind tunnel, ETW, model support, support correction

\section{INTRODUCTION}

The European Transonic Windtunnel (ETW) is a transonic cryogenic facility with a Mach number range up to 1.3 , pressurized up to $450 \mathrm{kPa}$, and can be cooled down to $110 \mathrm{~K}$, in order to reach Reynolds numbers of up to 50 million for full models [1]. In order to avoid blockage at transonic wind speeds, the top and bottom walls are slotted and allow the flow to deviate into a plenum chamber. Although the ETW has rather low interference and can deliver very precise data, further improvement of accuracy [2] is always sought for, and one method to achieve this is to find improved correction methods.

Correction methods for transonic wind tunnels are difficult to derive for various reasons. First, the slotted wall effect cannot yet be determined with sufficient precision. Although some attempts have been made, the results are not precise enough to make clear statements on slotted wall interference. Secondly, the different factors affecting the flow around the model cannot - as it is usually done for incompressible flow - be superimposed linearly. Instead, they interact in a non-linear way, and with rising Mach number and growing areas of supersonic flow, it becomes increasingly important to find an 'all-in-one' approach that reduces the amount of single factors used for the correction process.

Computational fluid dynamics (CFD) has the advantage of being able to calculate the complete transonic flow, thus reducing the need for superposition of different corrections. Although the modelling of slotted walls still poses a problem, support effects can be analysed well, because the need for differentiating between near and far field effects (and anything that might be in between) is removed, and it is possible to catch all aspects of support interference in one calculation.

The DLR project ForMEx (Fortschrittliche Methoden zur Extrapolation von Windkanalergebnissen auf den Freiflug - advanced methods for extrapolating wind tunnel results to free flight conditions) was initiated in 2003 in order to investigate how CFD can help to improve correction methods for wind tunnels, subsonic or transonic. The work described in this article was part of the project ForMEx.

The CFD computations described in this article were conducted using the DLR TAU code [3] on unstructured hybrid grids generated with Centaur ${ }^{\mathrm{TM}}[4]$. 


\section{AIMS AND OBJECTIVES}

The aim of the project is to create a simplified model of the ETW test section [1], in order to evaluate the influence of different support types on measurement results. The focus is on having a model as simple as possible, while capturing all effects of the model support precisely enough to compare and improve different support types and to gain knowledge on the mechanisms of support interference. Another aim is to investigate whether such a simple model can deliver improvements to correction methods.

\section{SIMPLIFICATIONS}

The question to what extent the tunnel geometry should be simplified was guided by several considerations. The resulting grids should be medium-tosmall sized, enabling the computation of several (Mach number and angle of incidence) polars with in-house resources, meaning grid sizes of well below 10 million points. Also, the boundary conditions should be well defined. This means that it should be easy to identify the influence of the support and to distinguish it from other effects, originating, for example, in a simplified wall model. The third point is that the model should be completed, validated, and used for the assessment of several support geometries within a 3-year time frame.

\subsection{Wall model}

Figure 1 shows the complete ETW test section including slotted walls, inlet nozzle, and plenum chamber.

The obvious method, to represent the complete geometry in one CFD grid, is too complex to achieve results within a 3-year time frame. Computations

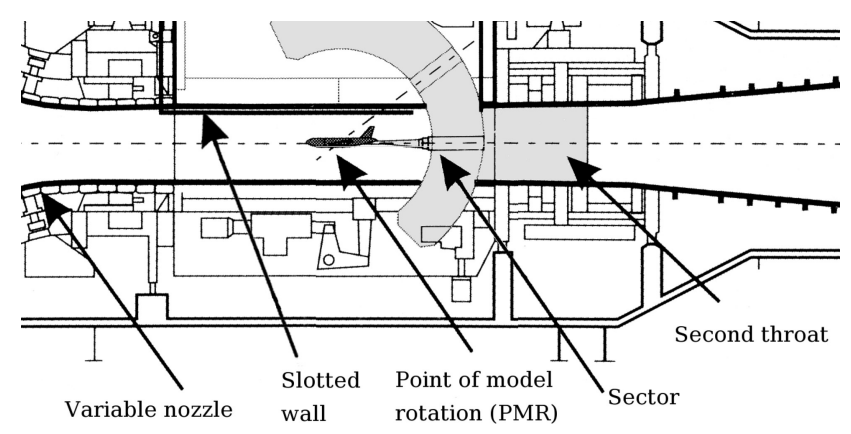

Fig. 1 Schematic side view of the ETW. The upper and lower walls of the test section are slotted and the whole volume inside the outer wall acts as a plenum chamber. The second throat behind the sector in the symmetry plane of the test section (source: ETW) with the complete tunnel geometry but with a simplified plenum of reduced size have been conducted in the HiReTT EU project [5]. Although there exist computational results, there are no properly validated results yet, as the slot wall flow is very complex and dominated by unsteady phenomena, separations, boundary layer effects, and secondary flow effects [6]. Thus, an unstructured NavierStokes grid properly representing the complete slot geometry and a plenum can be expected to have well beyond 10, probably exceeding 20 million nodes. Therefore, a direct representation of the slots is out of the question.

Another approach is to define a special 'slotted wall' boundary condition that allows throughflow, depending on the local wall pressure. Also here, several approaches are possible, some of which have been tried, but to the author's knowledge, none was successful. Also, a literature research delivered no results for successful implementations of such throughflow boundary conditions in Navier-Stokes codes. It was found that such an approach would consume too much time for setting up and calibrating, and it was decided to use a much simpler approach and leave the slotted walls out of the calculation.

The slotted walls in transonic wind tunnels are calibrated to minimize blockage. Thus, the effect they have on test results is much closer to free flight than to a solid wall. This is also reflected in the very small corrections applied at the ETW for slotted walls [2]. Actually, this means that a transonic computation with a far field and no walls comes very close to the real situation in ETW.

It would be possible to include the solid sidewalls in the computation, whereas the non-solid top and bottom walls are left out, although with some difficulties. The sidewalls would then need to be as large as the far field that replaces the slotted walls, which would require a lot of points in order to represent the boundary layer. Also, the nozzle could not be included correctly, which, in turn, would change the boundary layer on the sidewalls significantly. As there are very few experiments in the ETW involving a yaw angle, the final conclusion was that for the sake of simplicity, there should be no walls at all. This way it is also possible to have a defined environment for the assessment of support influence, without the need to differentiate between several interference sources.

\subsection{Representation of the support}

A view of the ETW model support mechanism is shown in Fig. 1. It consists of a circular sector whose centre point is called the point of model rotation (PMR). Rotating it around its centre 


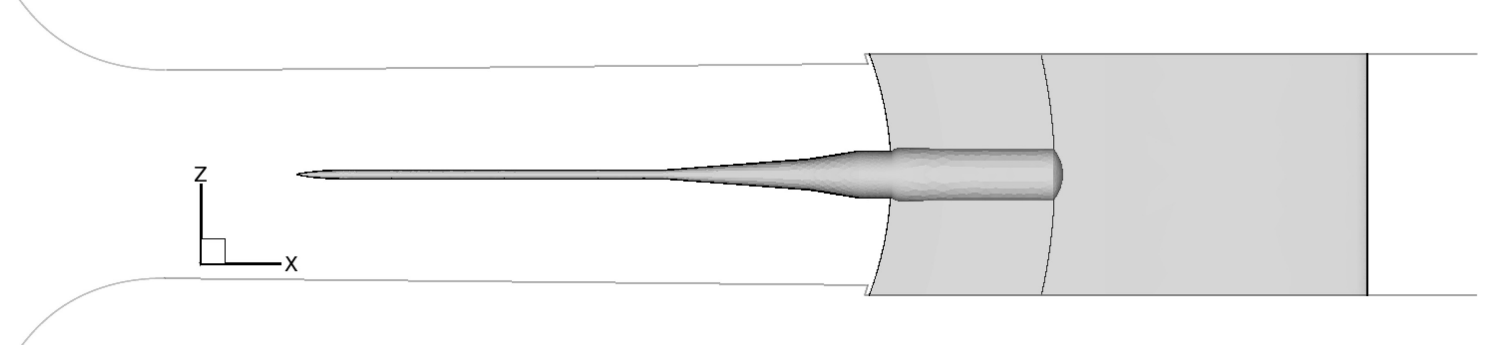

Fig. 2 The wetted parts of model support with short axial probe, mounted on the straight sting, plus second throat. Former wall locations are indicated by light grey lines

determines the pitch angle. On it is the sting boss, a bearing that alone allows for the roll movement of the model and in combination with sector rotation also allows yaw angles. The model rests on a sting for which different variants exist, e.g. straight, angled, twin, or fin sting. For calibration and the computations described in this article, only the straight sting was used.

Simply cutting out the part inside the tunnel test section would result in a geometry as shown in Fig. 2. The problem with this configuration is that the flow could pass the upper and lower ends of the sector. This would result in vertical flow components that are not present in the actual wind tunnel and would also lower the stagnation effect caused by the sector. To counter this, the sector was extended vertically as shown in Fig. 3. This was done by a straight extrusion of the sector profile at the point where the sector meets the tunnel walls and can also be understood as 'mirroring' the sector geometry. This way the effect of the sector meeting the walls behind the test section is included in the computations. Note that mirroring the complete support geometry would deliver a flow with no vertical components at the top and bottom wall locations over the complete length of the test section, thus representing the test section with closed top and bottom walls. By mirroring only the backward part, the solid walls at the sector location are represented,

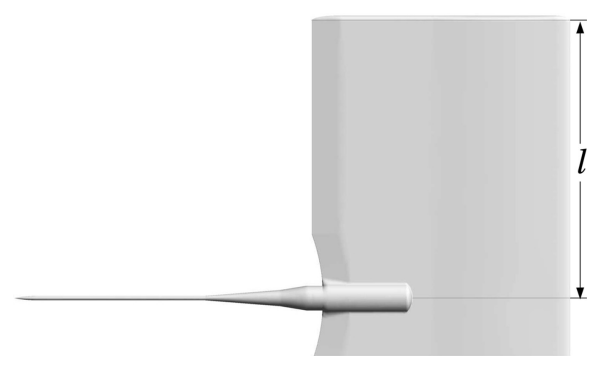

Fig. 3 The sector and second throat geometry are vertically extruded. $l$ marks the 'half span' of the resulting geometry and was part of a parametric study but further upstream, the flow can react to blockage in a similar way as in the wind tunnel with slotted walls. The extruded geometry was rounded off at the ends with continuous curvature to enable a smooth flow (and convergent CFD solution) around the ends.

The extrusion length $l$ (Fig. 3) was the object of a parametric study to determine the necessary length at which the upper and lower ends of the extended sector have no influence on the flow around the model. The results are presented in section 4.1.

In the process of preparing the available CAD geometry, a few details were added in order to make convergence easier and to represent the actual model support more precisely. This included rounding off the transition between sting boss and sector, which had formerly been a sharp angle. Also, the resulting CAD model is parametric, allowing for a quick change, for example, of the angle of incidence.

\subsection{Far field}

A spherical far field was used with the PMR at the centre. The radius was set to $25 \mathrm{~m}$, typical model half wing spans being $0.7-0.8 \mathrm{~m}$, to allow for sufficient distance from the extruded sector geometry. Although the flow around the further ends of the extended sector does not have to be modelled very precisely, it should still be fairly undisturbed by the far field boundary condition.

As only symmetric flight conditions are to be investigated and the test section itself is symmetric, only one half of the test section needs to be represented in the grid when using a symmetry plane in the $x-z$ plane.

In the ETW, the flow characteristics are adjusted such that inside the test section at a reference position at the wall, a dynamic pressure and temperature is reached that corresponds to the wanted Mach and Reynolds numbers at the model position. This correlation has been determined experimentally during the wind tunnel calibration [2]. 
Owing to the model support causing a slowdown of the flow in the model vicinity, far field values in the computation and flow conditions at the model point are also not the same. Because the ETW reference point is not present in the CFD grid (being on a wall that has been eliminated in the CFD geometry), it is necessary to establish a similar correlation that links far field values with corresponding values at the model location. This has been done using an iterative approach (section 4.2).

\section{CALIBRATION}

In order to achieve good consistency with actual ETW measurements, two parameters needed to be fixed: the minimum extrusion length of the sector and the far field Mach number corresponding to a model Mach number.

\subsection{Extrusion length}

A parametric study was made using the straight sting with an axial probe mounted on it (short axial probe (SAP)). Several lengths were applied and a calculation run with far field conditions set to $M=0.4$ and $R e=4.43 \times 10^{6}$. Figure 4 shows a CFD grid used in the study.

The incompressible Mach number was chosen as a worst case scenario. The upstream influence of the upper and lower ends of the extruded sector decreases with Mach number going towards 1; therefore, a length determined in an incompressible setting will also be sufficient in compressible flow.

For the extrusion length $l$, the values of $3,4,5$, and $7 \mathrm{~m}$ were used, each time the Mach number at the vicinity of the rotation point (PMR) was compared with the far field value. The aim is to find the extrusion length beyond which a further
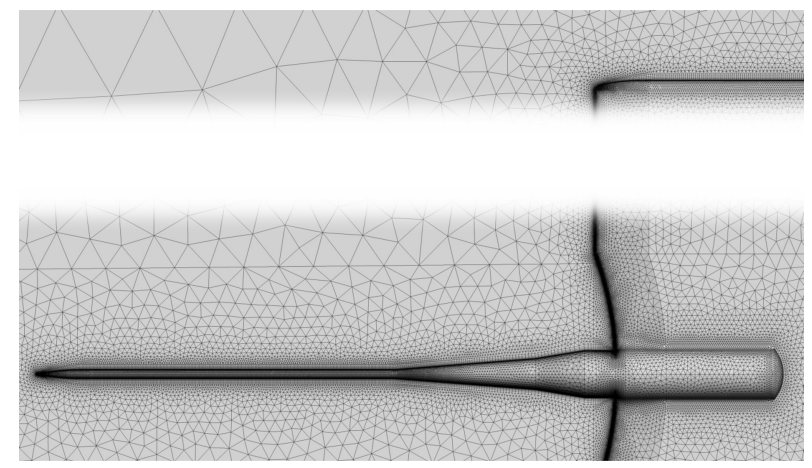

Fig. 4 CFD grid used in the parametric study of sector length ( $7 \mathrm{~m}$ in this case, 3.5 million points). The straight part of the extruded sector has been cut from the image increase would not change the Mach number at the model location.

In Fig. 5, the difference between far field and model Mach number is plotted against the extrusion length $l$. As can be seen, the influence is almost constant at an extrusion length of $7 \mathrm{~m}$ or more. Consequently, $l$ was fixed at $7 \mathrm{~m}$.

Later, compressible calculations confirmed that the influence of the finite sector is reduced at higher Mach numbers.

\subsection{Mach number correlation}

As can be seen, the difference between the far field Mach number and the Mach number around the model position is quite large, especially for higher values. It is thus clear that in order to obtain flow conditions at the model comparable with an experiment, a corresponding flow condition at the far field has to be found, similar to the real wind tunnel experiment [2].

In order to achieve this, a series of experiments in the ETW for which pressure data are known were replicated using the simplified representation. Again, the SAP configuration was used, as there are experimental data available for this setup, generated during the calibration phase of the ETW.

Using the same total conditions $\left(P_{\mathrm{T}}, T_{\mathrm{T}}, R_{\mathrm{T}}\right)$ as in the experiment, the Mach number at the far field was varied in order to reach the same static pressure at the PMR, which is roughly in the middle of the cylindrical part of the SAP, thus has practically undisturbed flow with the SAP mounted. The iterative algorithm applied for finding the appropriate Mach number uses the assumption that the necessary change in the far field Mach number was similar to

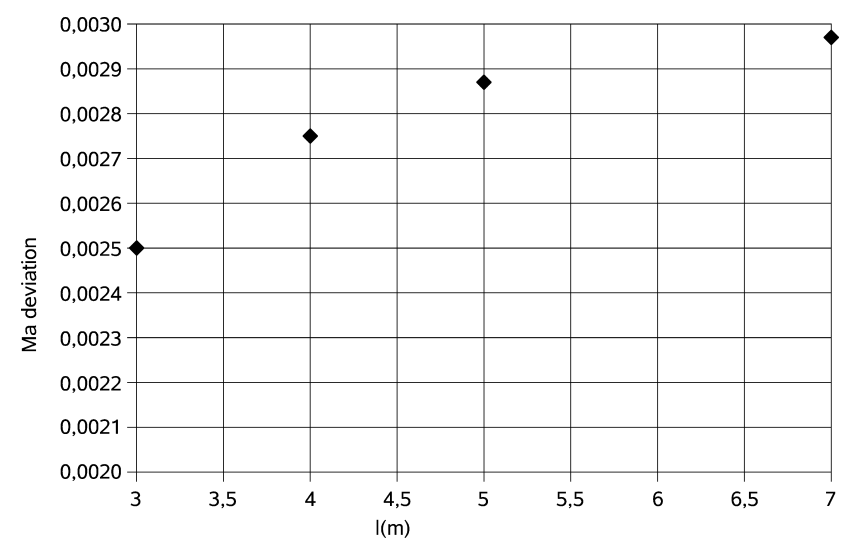

Fig. 5 Mach number difference between far field and centre of model rotation (PMR), depending on the length of the extruded sector. Extending the sector beyond $7 \mathrm{~m}$ will not change the conditions at the PMR significantly 
Mach no. difference between reference and PMR

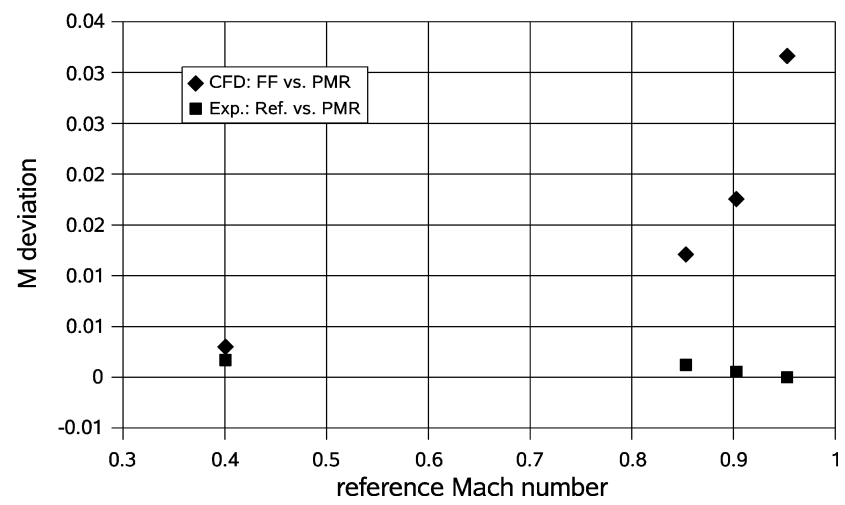

Fig. 6 Correlation between far field or reference Mach number and the isentropic Mach number at the PMR. At high Mach numbers, the difference between model and far field Mach number increases

the Mach number deviation at the PMR. Thus, for a given measured pressure at the PMR and at a given iteration step $i$

$$
M_{\mathrm{FF}, i+1}=M_{\mathrm{FF}, i}+\omega \cdot\left[M\left(P_{\mathrm{PMR}, \text { measured }}\right)-M\left(P_{\mathrm{PMR}, i}\right)\right]
$$

with a relaxation factor of $\omega=0.9$ and $M(P)$ being the isentropic Mach number corresponding to the constant total conditions and the measured/computed pressure at the PMR

$$
M(P)=\sqrt{\frac{2}{\kappa-1}\left[\left(\frac{P}{P_{\mathrm{T}}}\right)^{(1-\kappa) / \kappa}-1\right]}, \kappa=1.4
$$

With $P_{\mathrm{PMR}, i} \rightarrow P_{\mathrm{PMR}, \text { measured, }}$ the change in the far field Mach number becomes zero, and the wanted result is reached. This usually takes five to six iteration cycles. The resulting Mach number correlation as determined using the straight sting and the SAP is shown in Fig. 6.

As can be seen, the necessary correction to far field conditions is increasing with Mach numbers going towards 1 . Thus, to achieve $M=0.95$ at the PMR, a far field Mach number of about $M_{\mathrm{FF}}=0.98$ is needed.

This demonstrates the boundaries of the described wind tunnel model. Because the limit for the far field Mach number is 1 and the flow is slowed down in front of the sector, the highest Mach number attainable at the PMR is about 0.96 .

Further computations featuring a model on the support and with the same model in a far field without support show that the flow around a model on the straight sting support shows the same Mach number characteristics as on the same model without support, when using the model Mach number determined with the SAP geometry as a far field value (Fig. 7). Thus, it is a valid approach to use the Mach number correlation that was determined with the SAP geometry in order to determine the necessary far field Mach number for the 'model plus straight sting' geometry. For a different support, a new Mach number correlation has to be determined using the described method.

\section{COMPARISON WITH MEASUREMENTS}

For the case of $M_{\text {Ref }}=0.85$ and $R e=4.19 \times 10^{6}$, a comparison between experiment and CFD calculation was done, using the same SAP geometry that was used for calibration. Figure 8 shows the pressure distribution along the SAP and the conical part of the straight sting. At the PMR $(x=0)$, the measured and computed pressures match exactly, as this was part of the calibration. Apart from that, although, in general, both curves fit well together, a small gradient can be observed in the computed pressures, which is not present in the measured data.

The reason for this is that during the ETW calibration phase, the re-entry flaps and the wall angle were set to deliver the lowest possible axial pressure gradient. This means that in the ETW, the walls and re-entry flaps compensate a support effect that is visible here, namely, the slowing down of the oncoming flow ahead of the sting boss and the sector.

Because this gradient is indeed an effect of the model support, it is correctly represented in the computation, but the lower drag (due to buoyancy) and reduced $C_{\mathrm{L}, \max }$ (because the gradient promotes flow separation) due to this gradient will not be measured

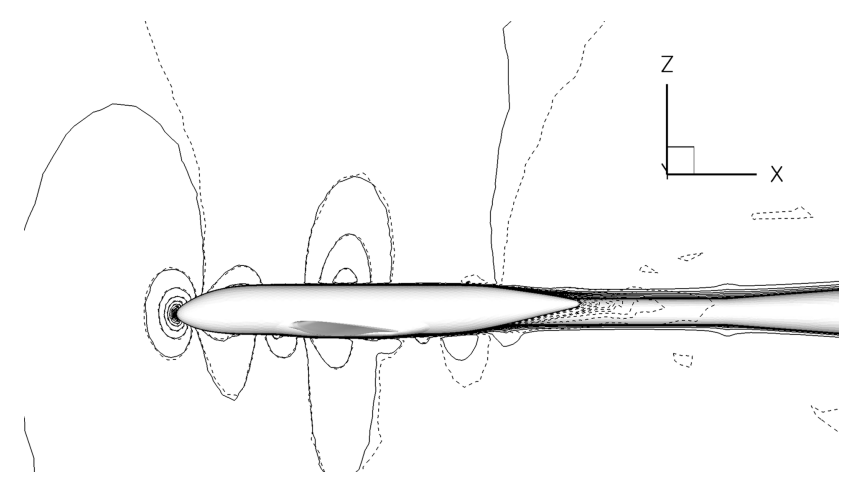

Fig. 7 Mach number contours of a wind tunnel model. Full lines: model mounted on straight sting; dotted lines: model without support, with the far field Mach number set to model Mach number, according to Fig. 6. Mach contours at the model centre coincide, whereas they differ at the rear end because of support interference 


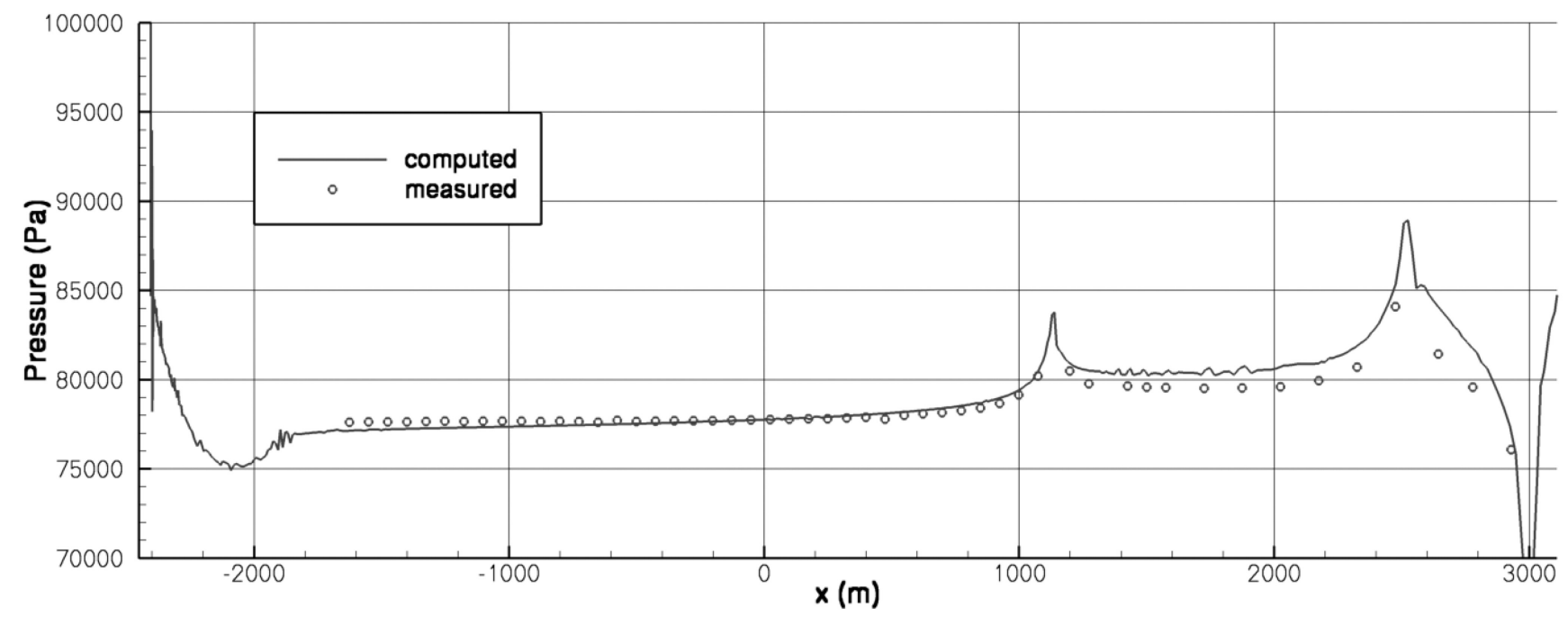

Fig. 8 Comparison between computed and measured pressure data for the case of $M_{\text {ref }}=0.85$ and $P_{\mathrm{T}} 125 \mathrm{KPa}$, along the SAP and the conical part of the straight sting. The PMR is at $x=0 \mathrm{~mm}$ (measured data: ETW)

in the actual wind tunnel, where the effect is not present.

It has to be concluded that without taking into account that the actual wind tunnel walls neutralize this gradient, a precise correction procedure cannot be derived using a far field model. However, the comparison of different support types and the investigation of support interference mechanisms are not influenced by this fact. Also, because the additional buoyancy coming from a known constant gradient can be easily subtracted from the results, a comparison with experiments is possible at least for cases where $C_{\mathrm{L}, \max }$ is not reached.

\section{CONCLUSION AND FUTURE WORK}

A simplified CFD model has been developed which represents the ETW model support inside a far field. With a typical model mounted on the support, a CFD grid contains roughly 4-5 million nodes and is thus suited for relatively fast computations of wind tunnel test cases.

Although a more accurate wall representation would be necessary to derive a proper correction method for the complete wind tunnel, support effects are easily determinable and different supports and support types can be compared and assessed. The Mach number correlation that was determined allows for replicating wind tunnel experiments with a straight sting in the CFD model, and the method is applicable to all kinds of supports.

Further investigations using a transport aircraft model are ongoing work, including polars, different Reynolds numbers, and different support types (e.g. twin sting). It is also planned to use the described approach as means for optimizing a new twin sting geometry within the EU project FLIRET.

\section{ACKNOWLEDGEMENT}

The author wishes to thank Dr Georg Wichmann, DLR, for managing the project ForMEx and for his support in writing this work.

\section{REFERENCES}

1 Walter, U. ETW user guide, January 2004, rev. A; ETW/D/ 95001/A, (European Transonic Windtunnel (ETW) $\mathrm{GmbH}, \mathrm{Köln})$.

2 Quest, J. Tunnel corrections in ETW. Technical memorandum ETW/TM/99024, ETW, Köln, March 1999.

3 Gerhold, T., Friedrich, O., Evans, J., and Galle, M. Calculation of complex three-dimensional configurations employing the DLR- $\tau$-code. AIAA 97-0167, 1997.

4 CentaurSoft $^{\mathrm{TM}}$ homepage, available from http:// www.centaursoft.com

5 Collercandy, R., Marquez, B., Lory, J., Dbjay, S., and Espiau, L. Application of CFD for wall and sting effects. HiReTT report HIRETTTNAFRCoWP2.231102003, Airbus France, October 2003.

6 Gazkov, S. A., Gorbushin, A., Ivanov, A., Semenov, A., Vlasenko, V., and Quest, J. Numerical and experimental investigations of slot flow with respect to wind tunnel wall interference assessment. AIAA-2004-2308, 2004. 


\section{APPENDIX}

\section{Notation}

$l \quad$ extrusion length of the sector (m)

$M \quad$ Mach number

$M_{\mathrm{FF}} \quad$ far field Mach number

$M_{\text {ref }} \quad$ reference Mach number

$\begin{array}{ll}P_{\mathrm{T}} & \text { total pressure }(\mathrm{Pa}) \\ R e & \text { Reynolds number } \\ R e_{\mathrm{T}} & \text { total Reynolds number } \\ T_{\mathrm{T}} & \text { total temperature }(\mathrm{K}) \\ & \\ \kappa & \text { isentropy coefficient (1.4) } \\ \omega & \text { relaxation factor }\end{array}$

